Sociedades Precapitalistas, vol. 8, nº 1 , e032, diciembre 2018. ISSN 2250-5121 Universidad Nacional de La Plata.

Facultad de Humanidades y Ciencias de la Educación.

Centro de Estudios de Sociedades Precapitalistas (CESP)

\title{
Cuestionando el concepto de redistribución en Creta Neopalacial: un aporte desde los sistemas de sellado
}

Jorge Cano Moreno

Centro de Estudios de Historia de Antiguo Oriente

(Universidad Católica Argentina) - CONICET, Argentina

Cita sugerida: Cano Moreno, J. (2018). Cuestionando el concepto de redistribución en Creta Neopalacial: un aporte desde los sistemas de sellado. Sociedades Precapitalistas, 8(1), e032. https://doi.org/10.24215/22505121e032

Recibido: 09 septiembre 2018 - Aceptado: 17 noviembre 2018 - Publicado: 07 diciembre 2018

(c) (i) (5) (2) Esta obra está bajo licencia Creative Commons Atribución-NoComercial-CompartirIgual 4.0 Internacional http://creativecommons.org/licenses/by-nc-sa/4.0/deed.es_AR 


\section{Cuestionando el concepto de redistribución en Creta Neopalacial: un aporte desde los sistemas de sellado}

Challenging the Redistribution Concept in Neopalatial Crete: a Contribution from the Sealing System

Jorge Cano Moreno

Centro de Estudios de Historia de Antiguo Oriente

(Universidad Católica Argentina) - Consejo Nacional de

Investigaciones Cientificas y Técnicas, Argentina

\section{Resumen:}

La redistribución es un concepto usualmente utilizado para explicar las relaciones económicas en las sociedades sin mercado o donde éste tiene una presencia más bien marginal. En el caso de la arqueología minoica, dicha teoría pareció encontrar un marco prolifero de aplicación dado que, desde las investigaciones de Evans, se consideraba que los 'palacios' concentraban todos los aspectos de la sociedad incluyendo la economía. Sin embargo, a partir de la década de los ochenta con el surgimiento de nuevas posturas teóricas en la arqueología, muchas de las categorías de análisis clásicas entraron en crisis y los 'palacios' fueron despojados de su poder monopólico dentro de los estudios de la cultura minoica. En este contexto el análisis de los sellos y de sus funciones administrativas y económicas pueden servir para clarificar hasta qué punto se puede hablar de redistribución en la cultura minoica. Palabras Clave: Redistribución, Cultura Minoica, Creta, Edad de Bronce.

\section{Abstract:}

Redistribution is a concept usually used to explain economic relations in non-market societies or where market has a rather marginal presence. In the case of Minoan archaeology, this concept seems to find a prolific framework of application given that, from Evans' investigations onwards, it was considered that the 'palaces' concentrated all aspects of society, including the economy. However, starting in the 1980s with the emergence of new theoretical positions in archaeology, many of the classical analysis categories went into crisis and the 'palaces' were removed from their monopoly power in the Minoan culture studies. In this context, the analysis of seals and their administrative and economic functions can serve to clarify to what extent it is possible to call redistributive the economic relationships in the Minoan culture.

KeYwords: Redistribution, Minoan Culture, Crete, Bronze Age.

\section{INTRODUCCIón}

Cualquiera que realice una primera aproximación a la sociedad de la Edad de Bronce ${ }^{1}$ en la isla de Creta (historiográficamente conocida como "Cultura Minoica” [Karadimas y Momigliano, 2004]) podrá observar que los denominados 'palacios' ${ }^{2}$ ocupan un lugar predominante en casi todas las explicaciones históricoarqueológicas. En este sentido, se ha llegado a conformar una imagen centrada en dichas estructuras como jerárquicamente hegemónicas en todos los aspectos sociales, políticos, religiosos, artísticos, administrativos y - como analizaremos en este artículo- económicos. Como bien ha señalado Molloy (2012), la tradición ha devenido en un "palacio-centrismo" a la hora de analizar la sociedad minoica (94).

Podemos encontrar en Evans al máximo impulsor de estas posturas; básicamente, el descubrimiento del 'palacio' de Cnosos hacia 1900 parecía mostrar que Creta había estado gobernada por un rey análogo a Minos tal y como lo establecían los relatos míticos. De hecho, su obra más importante, (The Palace of Minos at Knossos) publicada entre 1921 y 1935, vinculaba sus hallazgos directamente con la evidencia literaria clásica.

Poco importó que en los años sucesivos nuevos 'palacios' se hayan desenterrado (Malia y Festo, por ejemplo). A pesar de la importancia de estos descubrimientos, Cnosos seguía siendo el más grande y, según la mentalidad de la época, el de mayor jerarquía (Vavouranakis, 2007: 266). De este modo, el modelo general 
de Evans sabía adaptarse a prácticamente cualquier descubrimiento, siempre y cuando no se encontrara una estructura mayor, toda la evidencia se podía ordenar en la misma escala jerárquica.

Desde el punto de vista económico, los grandes almacenes de los 'palacios' parecían ser una evidencia suficiente para mostrar la importancia que habían tenido estos edificios en el control y la movilidad de bienes, ya sea dentro de la isla como fuera de ella (Alexiou, 1987: 252). De todas formas, estas aproximaciones no contaban con explicaciones que señalaran los distintos mecanismos de acumulación de bienes, por lo tanto, se consideraba que las materias primas llegaban al 'palacio' y allí se elaboraban productos manufacturados para ser intercambiados en el exterio. Sin embargo, como explica Lindgren (1987: 42), las preguntas para quién, cuánto, con qué sentido (y nosotros agregamos, cómo y por qué) quedaban sin responder.

No es hasta los trabajos de Finley que se estructuró una teoría general que pudiese abarcar las diferentes incógnitas que quedaban sin resolver. El historiador norteamericano aplicó las ideas de Polanyi (1944: 44-59) quien había establecido que en las sociedades antiguas primaban las relaciones de reciprocidad y redistribución. Según el escritor austríaco (p. 51), en la redistribución es clave la capacidad de almacenaje de los centros dado que permiten acumular los bienes que después se darían en forma de regalos (en los festines, por ejemplo) o de intercambios.

A su vez, parte de la explicación de Finley estaba influenciada por el desciframiento de las tablillas de Lineal $\mathrm{B}^{3}$ realizado por Chadwick y Ventris que, en su gran mayoría, correspondían a los archivos de los palacios micénicos. Evidentemente, estos documentos expresaban los pormenores de la administración palacial y de los territorios que le pertenecían y, por esta razón, daban una visión muy centralizada de la economía y de la sociedad (Halstead, 2011: 229-230). Así, Finley (1957: 135) sostenía que la economía micénica era "masivamente redistributiva" generándose un movimiento de bienes desde los almacenes del palacio a sus dependencias.

Dicha afirmación tendría un especial asidero en el área egea luego del estudio de Renfrew (1972) que intentaba explicar la "emergencia de la civilización" aplicando causas endógenas y sin recurrir a modelos difusionistas. Según este autor, los estados del Egeo - lo que incluye a Creta— habían nacido por medio de las prácticas redistributivas produciendo una integración vertical y horizontal (Halstead, 2011: 230; Branigan, 1988).

Considerando lo anterior, en este trabajo revisaremos la utilización del concepto de redistribución durante el período Neopalacial, el cual es usualmente tomado como el "apogeo" o "la edad de oro" de la cultura minoica (Adams, 2006: 26-27.). Para esto, nos sumaremos a las críticas realizadas por diversos autores y aportaremos nuevas ideas que relativizan el poder de los "palacios" minoicos haciendo hincapié en la economía a través del estudio de los sellos como elementos de control administrativo y burocrático.

\section{MODELO(s) EN CRISIS}

La narrativa histórica sobre la isla de Creta realizada por Evans comenzó a ser cuestionada luego del final de la Segunda Guerra Mundial. No fue desde la arqueología -dado que Creta fue campo de batalla durante este conflicto- de donde surgieron las críticas a lo que era una verdadera ortodoxia, sino desde la filología. Starr (1955), por ejemplo, cuestionaba la utilización de los mitos para establecer marcos interpretativos y conceptos como el de talasocracia. Si bien dichos argumentos podían ser eliminados o, al menos, matizados por provenir de otra disciplina ajena a la arqueología, lo cierto es que atacaban al corazón mismo de las justificaciones argumentativas de Evans, quien había basado sus posturas interpretativas desde los relatos literarios. De todas formas, si Evans no había necesitado la evidencia material para justificar sus afirmaciones, la falta de objetos en los que se pudieran apoyar sus críticos, sí que significaba una falencia metodológica y, por lo tanto, sus observaciones eran descartadas y prácticamente abandonadas.

Reestablecidas las excavaciones en Creta, los sucesivos descubrimientos y las nuevas teorías procesuales en la arqueología que demandaban más rigor metodológico y teórico en los investigadores y en sus 
interpretaciones generaron una revisión en las posturas establecidas desde la obra de Evans. Repasemos tres obras centrales a modo de ejemplo que son actas de congresos realizados con anterioridad a su publicación, The Minoan Thalassocracy Myth and Reality (Hägg y Marinatos, 1984), The Function of the Minoan Palaces (Marinatos y Hägg, 1987) y The Function of the "Minoan Villa"(Hägg, 1997). En ellas, distintos académicos presentaron ponencias que apoyaban, dudaban o cuestionaban los enfoques clásicos. No reseñaremos estas obras dado que no es la intención de nuestra publicación, pero si podemos mencionar que utilizaremos muchos de los trabajos que las integran a lo largo de nuestra argumentación. Obviamente, esto no quiere decir que no haya habido otras publicaciones individuales que cuestionaran o pusieran en duda la ortodoxia reinante, pero lo importante de estas obras colectivas es que demuestran que existía la necesidad de revisar las bases de la narrativa general iniciada a principios del siglo $\mathrm{XX}$, aunque - como es lógico suponerpocas veces, sino nunca, se llegó a una conclusión. Sin embargo, el simple hecho de que se hayan formulado diversas preguntas y cuestionamientos implica, en cierta medida, que epistemológicamente se comenzaba a resquebrajar el modelo reinante.

Ya en los años cercanos al advenimiento del siglo XXI y en los años posteriores a éste, las teorías postprocesuales hicieron un especial énfasis en analizar los marcos epistemológicos, teóricos y socialesculturales para explicar aquello que subyacía a las al modelo de Evans y sus continuadores en el presente. Más allá de que muchos de los arqueólogos e historiadores que mencionaremos en este trabajo no subscriban o no se reconozcan como postprocesuales, hay que admitir que la gran mayoría de ellos parten de una base que podríamos clasificar como 'deconstruccionista' en cuanto que dedican sus introducciones al análisis de ciertos conceptos establecidos acríticamente. Esto toma una especial relevancia en los estudios de Creta durante la Edad de Bronce ya que la gran mayoría de conceptos (iy hasta el mismo nombre de minoicos!) son términos construidos historiográficamente y, a la vez, marcos interpretativos.

Otro de los puntos importantes a analizar fue el continuo descubrimiento de grandes estructuras que no poseían una Corte Central (Central Court ${ }^{4}$, pero que sí contaban con elementos arquitectónicos similares al de los 'palacios'. Nos referimos a las 'villas' cuya presencia en el registro arqueológico de la isla es mayoritaria. De hecho, su presencia parece ser caprichosa: algunas cercanas a los 'palacios', otras en núcleos urbanos, un buen número en territorio agrícola y otro tanto sobre la costa o en zonas montañosas (Betancourt y Marinatos, 1997).

Como es posible ver en la bibliografía clásica, estas estructuras fueron interpretadas, genéricamente, como territorios satélites de los 'palacios' y, en última instancia, de Cnosos. Pero también, la misma información ha sido utilizada como la base argumentativa para sostener la regionalización de entidades políticas más o menos independientes (Rehak y Younger, 1998: 106; Christakis, 2008). En esta línea, las funciones de estas estructuras - especialmente, las económicas y las administrativas - son un punto central para analizar si podemos hablar de redistribución en los 'palacios' y en la Creta minoica en general. Dichos cuestionamientos, también se traducen en una puesta en jaque del modelo jerárquico que ve en Cnosos y en los restantes 'palacios' como el centro de la hegemonía.

Un último punto interesante respecto al tema de la redistribución es que dicho concepto fue utilizado para argumentar la existencia de reyes en los 'palacios' minoicos. Si tomamos como ejemplo el trabajo de Betancourt (2002: 211) notaremos que las capacidades redistributivas de estas estructuras para suplir las necesidades de la población en tiempo de crisis y mantener a su población estable son tomadas como probatorias de la existencia de un líder análogo al rey y, por lo tanto, de la jerarquía de los 'palacios' en todos los aspectos sociales.

\section{Redistribución y poder en Creta Neopalacial}

Como establecimos, en el modelo clásico, la economía redistributiva formaba parte de las funciones de los 'palacios' y de su hegemonía sobre distintos aspectos sociales. Por lo tanto, fue imprescindible para los 
distintos arqueólogos y académicos mostrar la importancia del control sobre la economía que tenían los administradores de estos centros. En este aspecto, se cruzan los dos elementos centrales de nuestro análisis dado que también los sellos y los sistemas de sellados están íntimamente ligados con la burocracia económica.

Hay dos cuestiones que podemos destacar en las bases argumentativas del sistema redistributivo, uno historiográfico y otro arqueológico. Respecto a la primera, es importante resaltar la influencia que tuvo el modelo de los estados orientales en los estudios minoicos. En resumidas cuentas, las ciudades mesopotámicas contaban con dos grandes centros redistributivos como los palacios y los templos que, según las posturas tradicionales, se encargaban de acumular excedentes con el fin de producir otros productos secundarios, mantener al aparato administrativo, político y religioso del estado y tener disponibles los bienes necesarios en caso de malas cosechas o carestías ${ }^{5}$ (Nakassis et al., 2011; Schoep, 2010). Adicionalmente, ya mencionamos el peso que tuvo el desciframiento de las tablillas de Lineal B y el modelo económico que de éstas se desprendía y, sobre todo, la suposición de que los archivos de Lineal A transmitan una información similar (Whitelaw, en prensa). En ambos casos, la redistribución parecía estar justificada desde las fuentes y los enfoques teóricos.

En el caso de la evidencia arqueológica, los 'palacios' ofrecen amplios espacios de almacenamiento con grandes pithoi y con piezas cerámicas de diferentes tamaños para el resguardo de bienes, principalmente, primarios. Este punto es fundamental dado que la acumulación de materias primas juega un rol decisivo en el modelo redistributivo, sobre todo, teniendo en cuenta que la población urbana debió haber dependido de manera directa de la producción agrícola rural por las dimensiones mismas de estos centros (Branigan, 2001: 48). En esta acción, se pone en juego, en primer lugar, la capacidad y el poder de las estructuras centrales para adquirir estos productos; esta situación tan solo la podemos plantear en términos hipotéticos dada la falta de documentos escritos. En segundo lugar, el flujo de bienes hacia los 'palacios' es, en este modelo, imprescindible para llevar a cabo una serie de estrategias económicas como el intercambio, la elaboración de materias primas, la manutención de los líderes religiosos y políticos y del personal dependiente de éstos; en definitiva, la movilidad de bienes es necesaria para darle vida a las prácticas redistributivas.

En este aspecto, los trabajos de Christakis (2004, 2008 y 2011) han demostrado dos interesantes tendencias respecto a los 'palacios' y su capacidad de almacenaje. La primera, el autor detecta cierta intencionalidad en aumentar los espacios destinados al acopio de bienes y de sus respectivos recipientes, éstos de diversa índole y de una gran variedad de tamaños (2004: 307). La segunda idea está relacionada con establecer si el aumento en el almacenaje de materias primas durante el período Neopalacial son un indicador fiable para evidenciar arqueológicamente prácticas redistributivas. Respecto a esta posibilidad, el autor es taxativo y luego de analizar la capacidad de almacenaje de los centros palaciales y de las 'villas' y la población estimada de los núcleos urbanos y rurales señala que la capacidad no era lo suficientemente grande como para alimentar a este número de personas, ya sea que vivieran dentro del 'palacio' o en sus alrededores (2008, passim). Por lo tanto, hay que considerar a la redistribución como una de las estrategias económicas del período -entre tantas otras- y, tal vez, sólo haya estado orientada a la acumulación de materias primas orientadas a los ritos religiosos (2011: 203-204). Este tipo de análisis tiene que ser considerado en otros aspectos que van más allá de la capacidad de adquirir bienes primarios, sino que implica también, relativizar la función de los 'palacios' como productores de bienes manufacturados y como actores principales del intercambio.

Respecto al primer punto, tradicionalmente se ha considerado a estos centros como los encargados de producir distintos bienes secundarios por medio de los talleres que se encontraban en su interior o controlando a talleres y artesanos dentro de su área de influencia (Marcelis, 1989: 32). Por ejemplo, Branigan (1983: 24; 29-30) señalaba la existencia de, al menos, tres tipos de artesanos: aquellos que trabajaban directamente vinculados con el 'palacio'; los que estaban vinculados a éste de manera parcial; y los artesanos itinerantes, los cuales eran más difíciles de distinguir en el registro arqueológico. De estos tres tipos, para el autor dos estaban intrínsecamente vinculados de manera total a estos centros y el último grupo era difícil imaginárselo fuera de la órbita de control de dichas estructuras. Este tipo de interpretaciones se encuentran dentro de una visión imperante y jerarquizada de los 'palacios' como controladores totales de 
la economía cretense durante la Edad de Bronce. De hecho, están totalmente relacionadas con el concepto de la redistribución en cuanto la producción de bienes secundarios a partir de materias primas es una de las maneras de distribuir recursos tras haber cumplido con la acumulación necesaria de materiales.

Sin embargo, las críticas sobre el modelo redistributivo también afectaron a este enfoque. No podía ser de otra manera ya que la adquisición, producción y distribución de bienes, según la postura ortodoxa, formaban parte del mismo sistema. En este sentido, Pullen (2010:3) en la introducción de Political Economies of the Aegean Bronze Age, destaca que las contribuciones de dicho volumen buscan comprender cómo la economía de varias sociedades funcionó dentro y fuera de los controles palaciales. Así, Driessen (2010) ha dedicado un largo capítulo a explicar la importancia de los hogares (bouseholds) en las relaciones políticas, económicas y religiosas en Creta mostrándolos como principales actores en la historia de la isla y Schoep (2010: 60; 73 y ss.) ha destacado la gran cantidad de talleres y producciones de productos de elite fuera de la órbita de los distintos 'palacios' y que incluso los talleres cercanos a estas estructuras tampoco controlaban el destino y el posterior uso de los bienes que elaboraban.

Respecto al segundo punto -los 'palacios' como controladores del intercambio- también se han generado serios cuestionamientos a este esquema (Kopcke, 1987: 260). La idea principal era que la movilidad de bienes se realizaba entre cortes reales siguiendo el ejemplo de Egipto, Mesopotamia y Siria-Palestina (Alexiou, 1987: 251). Hoy en día, la base interpretativa de dicha analogía no puede ser sostenida (véase Barjamovic, en prensa), así como también se ha destacado que uno de los grandes problemas está relacionado con la escala que decidamos utilizar para estudiar el intercambio ya que las esferas de influencia no pueden ser iguales en escalas locales, regionales y de larga distancia (Parkinson, 2010: 29). En esta línea, con sólo realizar un vistazo rápido a la bibliografía existente es posible relativizar la importancia de los 'palacios' en los contactos con otras entidades políticas (Melas, 1988; Knapp, 1990; Parkinson, 2010; Mcdonald, Hallager y Niemeier, 2009); a su vez, tampoco parecen haber dominado los intercambios regionales en el Egeo (Wiener, 1990 y 1991; Michailidou, 1999; Mountjoy y Ponting, 2000; Parkinson, 2010; Knappet y Nikolakopoulou, 2008); e, incluso, a nivel local, las diferencias regionales en la distribución de diferentes productos de alto valor social, también parecen indicar que los 'palacios' fueron un componente más en las relaciones de intercambio y, en muchas oportunidades, tampoco fueron los más importantes (Michailidou, 1999: 96-97; Schoep, 1999a y 1999 b y 2002).

Así y todo, podemos destacar que el gran debate en el aspecto económico son los modelos teóricos y los mecanismos de interacción social entre unidades políticas-sociales que se utilicen para analizar la evidencia arqueológica. De todas formas, la realidad parece ser mucho más compleja que los componentes analíticos que proponen los diferentes enfoques y cada día nos acercamos más a definiciones amplias que se muestran más flexibles a la hora de establecer un marco de referencia. Aun así, resulta claro que la redistribución ya no puede ser tomada en cuenta para representar la totalidad de las relaciones económicas (Earle, 2011) ya que los 'palacios' no parecen haber sido los centros acumuladores de bienes primarios y secundarios, así como tampoco tuvieron un protagonismo monopólico o, incluso, destacado, en las relaciones de intercambio.

\section{UN REPASO SOBRE LOS SISTEMAS DE SELLADO}

Por más limitado que pudo haber sido el sistema redistributivo minoico en el período Neopalacial, eso no implica que no haya tenido sus propios mecanismos de control para verificar el ingreso y egreso de distintos bienes. En este punto es donde la evidencia de los sellos y los sellados minoicos puede echar alguna luz sobre los centros administrativos de la isla y su capacidad de movilización de bienes. Este aspecto es sencillamente crucial dado que los sellos son el segundo elemento arqueológico más abundante en la historia de la isla de Creta sólo por detrás de los fragmentos cerámicos y que, adicionalmente, cuentan con la ventaja de estar catalogados casi en su totalidad en el Corpus of Mycenaean and Minoan Seals (en adelante, CMS). Con más 
de 10.000 ejemplares (Kryzcoskca, 2005: 1), la mayoría de estos materiales están asociados, sobre todo en el período Neopalacial, a centros territoriales de importancia y con cierta influencia en la movilidad de bienes ${ }^{6}$.

En esta sección, nos concentraremos en mostrar los diversos sistemas de sellados relacionados, posiblemente, con el ingreso y egreso de mercancías. Para esto, debemos tener en cuenta que por la naturaleza fragmentaria de la evidencia arqueológica sería demasiado arriesgado especular la cantidad de bienes controlados a través de los sellados dado que tan solo conocemos una pequeña cantidad de estas transacciones. Evidentemente, éstas solo se concentran en los intercambios relacionados con las estructuras monumentales en donde se han encontrado los sellos, las cuáles pueden ser divididas en 'palacios' y 'villas'.

Adicionalmente, el recurso administrativo del sellado estuvo ligado al registro escrito ya que diferentes tablillas en escritura Jeroglífica y en Lineal A fueron encontradas en los mismos sitios (Weingarten y Webb, 1994: 94). Estos vínculos parecen haber sido estrechos dado que muchos ideogramas (de acuerdo a algunas analogías con las descifradas tabillas de Lineal B) se refieren a bienes primarios y secundarios concretos, por lo cual los documentos escritos podrían estar controlando la entrada y la salida de estos recursos. Otro rasgo importante de la relación entre sellos y escritura es que algunos sellados parecen haber estado asegurando rollos atados de papel (fig. 1).

Figura 1. Marcas de cuerdas en los sellos. Tomado de Weingarten 1983: 9.
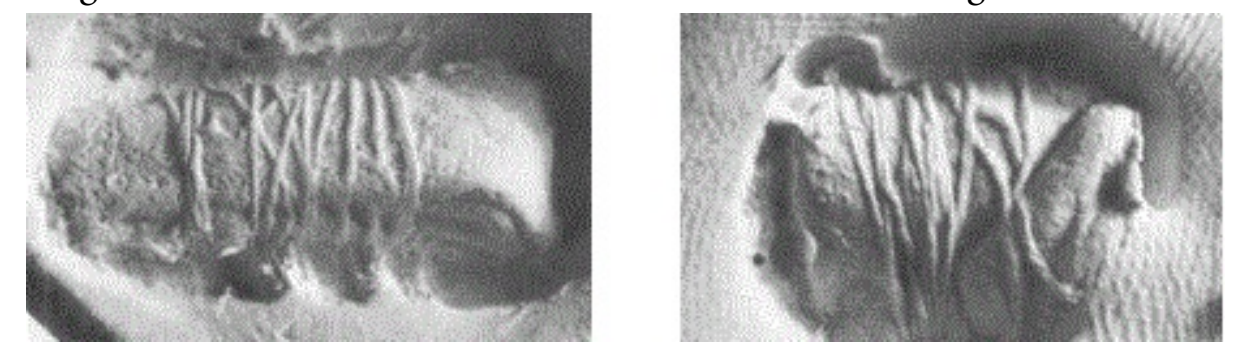

Es importante remarcar que, si bien lo sellos se pueden encontrar bien temprano en la historia cretense durante el Minoico Temprano II (Prepalacial), ca. 2600 a.C. (Crowley, 2013: 2), el desarrollo de los sistemas de sellado es posterior y está estrechamente vinculado con el surgimiento de los grandes centros de poder regionales (Weingarten, 1990a: 105-107). Así, en la historia cretense primero encontramos sellos que no forman parte de ningún sistema administrativo en particular, sino que más bien fueron usados como elementos de prestigio para señalar el estatus de sus dueños. Ésta, es una anomalía difícil de explicar y que escapa a los fines de este trabajo, pero que puede encontrar algunas respuestas en el carácter imitativo de prestigio que tuvieron los primeros sellos en Creta (Galanakis, 2005: 20).

Lo interesante en este punto es que los sistemas de sellado en la isla de Creta surgieron en el mismo período, pero de manera diferente. En otras palabras, cada centro adoptó una manera distinta de llevar a cabo sus controles, lo que generalmente se ha interpretado como una relativa independencia de un centro respecto a otro (Anastasiadou, 2006: 159-160). Si bien estas afirmaciones corresponden al periodo Protopalacial, es posible encontrar evidencia similar que indica un funcionamiento no centralizado en la burocracia a lo largo de la isla. En esta línea, expondremos a continuación una serie de diferentes casos estudiados que muestran la variedad en los sistemas de sellado.

En primer lugar, podemos mencionar el caso del 'palacio' de Festo, que es probable que haya sobrevivido sólo hasta los primeros años del periodo Neopalacial. En resumidas cuentas, el sistema de este centro se caracteriza por el sellado directamente sobre objetos relacionados con almacenes (Weingarten, 1986: 280.), aunque también la escritura Lineal A ya era utilizada, pero no dentro de estos espacios (Schoep, 2001: 90). De los objetos sellados, un $10 \%$ corresponde a piezas identificables, mayormente jarras o esteras, mientras que el $90 \%$ restantes son piezas de arcilla que se encontraban en perillas de puertas y clavijas de madera (Weingarten, 1986: 280; Schoep, 2001: 89; Hallager, 2001). A esta información, debemos agregar que unos 1289 nódulos 
parecen haber sellado solo 11 objetos de madera, por lo que los investigadores consideran que cada vez que se ingresaba o sacaba un bien del almacén, se marcaba dicho movimiento a través de un sello específico y que, incluso, pudieron llegar a conservarse los sellados rotos como evidencia de la transacción realizada a manera de archivo (Weingarten, 1986: 280; Schoep, 2001: 89). Por último, es importante destacar es que la mayoría de los sellados parecen provenir de una pequeña cantidad de sellos (Weingarten, 1990b: 72).

En el caso de Cnosos, el Temple Repositories, un poco posterior al yacimiento en Festo (MM IIIB, transición entre el Protopalacial y el Neopalacial), es el depósito que más sellados nos ofrece con alrededor de 160 especímenes (Galanakis, 2005: 57). Este sistema de sellado se conoce como Sistema de Sellado Múltiple (MSS, por sus siglas en inglés) y tiene ciertos puntos en común con el de Festo, pero con diferencias substanciales (Schoep, 2001: 91). En cuanto a las similitudes, es posible destacar que también se pueden encontrar una diversidad de sellados sobre una misma superficie, pero con la diferencia de que no se utilizó el mismo sello, sino que cada uno cuenta con diferentes motivos (Weingarten, 1989: 40). Finalmente, las piezas de arcilla cuentan con dos orificios que dan a pensar la posibilidad de que hayan servido para atravesar una cuerda y así, ser colgados de los objetos, remplazando el sellado sobre ellos (Schoep, 2001: 91-92). Además, se puede notar un abandono de los sellados con escritura Jeroglífica por los sellos con imágenes, lo que ha sido interpretado como el surgimiento de una nueva clase de oficiales burocráticos (Galanakis, 2005: 58).

En el extremo occidental de la isla tenemos a la ciudad moderna de Chania que se ubica exactamente en el mismo lugar en donde solía estar la urbe minoica. Por esta razón, es difícil saber ante qué tipo de estructura monumental nos encontramos. La evidencia sobre el sistema de sellado y la administración del sitio sigue siendo una molesta incógnita dada la gran cantidad de sellos y de tablillas en Lineal A que se han encontrado en las diversas excavaciones de rescate (Hallager, 1996: 48). Respecto a este último formato escrito, tan sólo Hagia Triada cuenta con más tablillas con este tipo de escritura, lo que demuestra la importancia administrativa del lugar (Krzyszkowska, 2005: 173).

La gran mayoría de los sellados se ubicaron sobre dos formatos: primero en cantidad, encontramos a los roundels, un tipo de documento administrativo especialmente utilizado para inscripciones de Lineal A (Hallager, 1996: 36-37), pero que puede llegar a tener un sellado en alguno de sus bordes (fig. 2), de hecho 2/3 del total de este tipo de sellado proviene de Chania; en segundo lugar, a los nódulos colgantes como en Cnosos y Festo (Krzyszkowska, 2005: 176). Respecto al primer tipo de sellado, es importante marcar que diferentes sellos podían servir para marcar distintos tipos de productos dado que la escritura así lo señala (Krzyszkowska, 2005: 176-177)

Además, se han encontrado otras dos formas de sellados muy particulares: una barra de arcilla con 12 sellados, de los cuales 10 son diferentes entre sí, lo que hace muy difícil saber su utilización; unos pequeños tapones de arcilla con sellos en la cima (fig. 3) que escapan a las categorías conocidas; y unos sellos que se ubicaban sobre los bordes de los pithoi (fig. 4) aunque aún no se puede conocer del todo su función (Hallager, 2001: 5-11). 
Fig. 2. Roundel con marcas de sellado. Tomado de Andreadaki-Vlasaki y Hallager, 2007: 14.
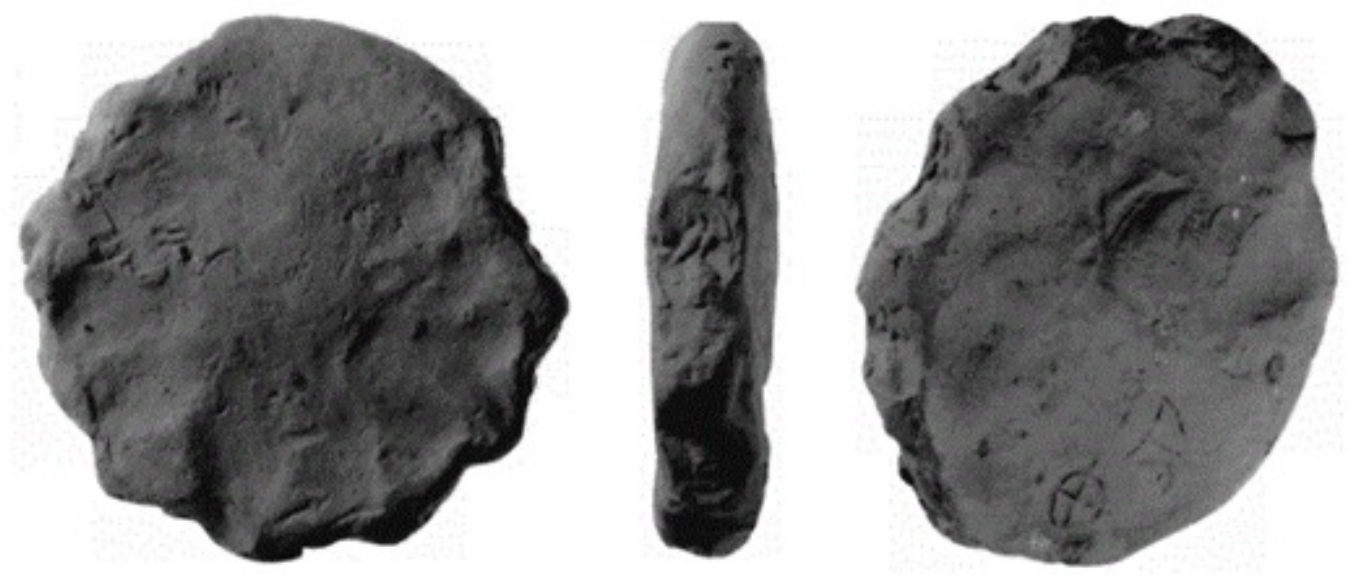

Fig. 3. Tapón de arcilla. Tomado de Hallager, 2001:6.

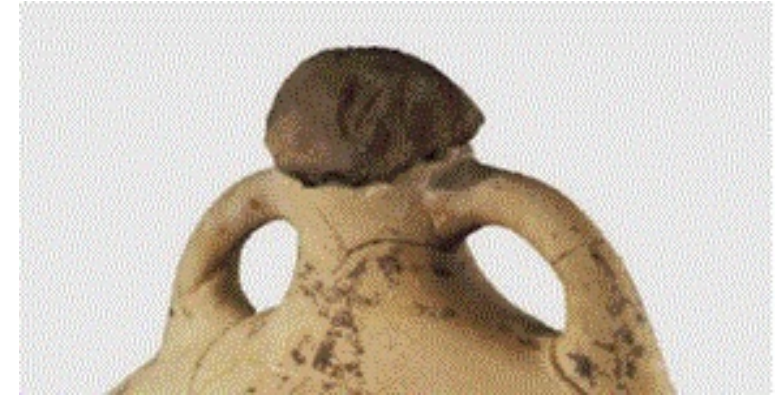

Fig. 4. Sello sobre Pithos. Tomado de Hallager, 2001: 9.

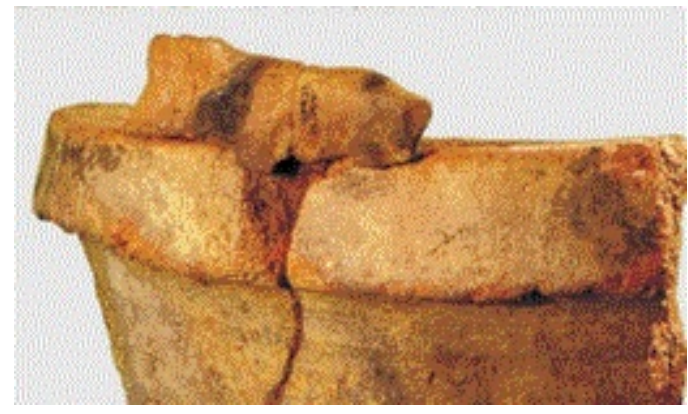

Por su parte, Zakros tiene la ventaja de haber sido descubierto mediante una intervención arqueológica moderna realizada en la Casa A, cercana al 'palacio'. El primer punto para destacar es que nuestro conocimiento del sistema administrativo de Zakros está constituido por unos 262 sellos que pudieron haber impreso alrededor de 548 nódulos que, a su vez, portan un total de 1005 sellados. En Zakros, por un lado, adoptan prácticas similares a las de Cnosos (el MSS y la combinación de diversos sellos), pero, por otro lado, portan un tipo de imágenes muy singulares que no tienen paralelo en el resto de la isla (Dionisio et al, 2014: 106; Weingarten, 1986: 289).

A sólo 3 kilómetros del 'palacio' de Festo se ubica la 'villa' de Hagia Triada, la más grande de las estructuras no palaciales de toda Creta. Éste es un sitio de gran importancia ya que, entre otras características, cuenta con una gran cantidad de sellos que constituyen el archivo más abundante de toda Creta durante el período Neopalacial con 146 tabletas de Lineal A y 1150 sellados (Galanakis, 2005: 66; Krzyszkowska, 2005: 168). 
De hecho, sellos y escritura parecen haber formado parte del mismo sistema administrativo en general y es muy difícil separarlos (Weingarten, 1987: 1-2).

En esta línea, hay que destacar que 847 impresiones fueron producidas por 17 sellos y que otros 127 sellos produjeron los 210 sellados restantes (Weingarten, 1987: 2-3). La gran mayoría de los nódulos presentan un solo sellado, por lo cual se lo ha denominado como Sistema de Sellado Simple (SSS, en inglés Simple Sealing System) (Weingarten, 1987: 15-20). Como el mismo sello podía dejar su marca en cualquiera de los formatos conocidos y como no se ha encontrado un patrón entre un sello y un determinado documento, se considera que las marcas de Lineal A eran una manera de verificar el contenido o las cantidades y que los sellos funcionaban como etiquetas del bien en sí, ya que 957 de estos sellados (Galanakis, 2005: 66 ${ }^{7}$ ) tenían un orificio para ser colgados (Weingarten, 1987: 7). En términos más generales, se ha establecido que la gran cantidad de sellos e inscripciones era una manera de controlar la producción de bienes en los talleres y su respectivo almacenaje en habitaciones preparadas para esa función.

Por otro lado, hay una buena cantidad de sitios que presentan una menor cantidad de sellos, ya sea porque las excavaciones no han sido lo suficientemente extensivas o porque tal vez no estemos ante un sistema de sellado organizado y centralizado, sino más bien una práctica reducida y esporádica.

Comenzaremos por el 'palacio' de Gournia, del cual no contamos con suficientes elementos para saber si había un sistema de sellado. De todas formas, sí podemos saber que una veintena de sellos provienen del 'palacio' (Hawes et al, 1908: 54) y otra pequeña cantidad se han encontrado en el poblado alrededor de éste (Watrous y Heimroth, 2011: 208). Lo que refuerza nuestra idea de que es posible que haya existido un sistema de sellado es que, además de sellos, se han encontrado tablillas con inscripciones de Lineal A como en Hagia Triada, Chania, Cnosos y Zakros (Watrous et al., 2015: 443-448). Sin embargo, por la cantidad y heterogeneidad de los sellos, los excavadores no han aventurado ningún tipo de sistema en particular (Soles, 1991:74-75). De todas formas, Younger considera que los sellos del periodo Neopalacial tienen una conexión muy fuerte con Cnosos, por lo que piensa que Gournia pudo haber sido un nexo que conectaba a este centro con la parte oriental de la isla y que, a cambio de los servicios prestados, Cnosos le brindaba algún tipo particular de bienes que estaba acompañado por sellos o sellados (Younger, comunicación personal, mayo 2017).

Otro sitio importante por su evidencia es la 'villa' de Sklavokampos en la cual se encontraron 39 sellados de los cuales 37 tenían múltiples sellados (MSS) (Galanakis, 2005: 62) que, en su mayoría, fueron realizados por anillos-sellos (Krzyszkowska, 2005: 187 ). Este yacimiento parece haber tenido un carácter rural, lo que significa que no se han encontrado otros yacimientos alrededor o que, de haberlos, son pequeños. Ante esto, desde nuestra postura, los sellados podrían estar indicando dos posibilidades que no se excluyen, sino que podrían complementarse: la primera es que el sistema de sellado — por más simple que haya podido serpodría haber servido para administrar la 'villa' emulando el sistema de sellado de Cnosos (Christakis, 1999: 15). La segunda posibilidad, y la más difundida en la bibliografía, es que estos sellos indican el intercambio de bienes entre sitios de importancia que, además de Cnosos, incluiría a Hagia Triada, Zakros y, tal vez, Acrotiri (Krzyszkowska 2005: 187-188).

A 6 kilómetros de Sklavokampos, se encuentra Tylissos, otra de las 'villas' importantes de Creta. En ambos casos, contamos con pocas evidencias del sistema de sellado dado que solamente hay cuatro sellos. Sin embargo, incluimos a esta estructura en esta sección ya que pareció tener algún sistema de almacenamiento fijo por la presencia de dos tablillas de Lineal A y porque el almacenaje se realizaba en una habitación especialmente preparada para esa función (Christakis, 1999: 11).

La última de las estructuras no palaciales que trataremos es el yacimiento de Pyrgos que tiene una evidencia similar a Tylissos en cuanto sólo se han encontrado cuatro sellos, pero que están acompañados de tabillas de Lineal A (Krzyszkowska, 2005: 186). También, el sitio presenta similitudes con Sklavokampos, ya que ambos parecen haber sido el centro de sus regiones más próximas (Christakis, 1999: 13), aunque es complicado asegurar los lazos de este sitio con los grandes centros de la isla (Knappet, 1999 passim). 
Palaikastro es otro de los lugares en los cuales se han encontrado una pequeña cantidad de sellos (media docena aproximadamente). La cuestión principal de tal falencia es que en este sitio aún no se ha encontrado el edificio central (probablemente un 'palacio'), sino que la mayoría de las excavaciones se han hecho sobre el poblado (MacGillivray y Sackett, 2010). De todas formas, los sellos y sellados conocidos se pueden relacionar con los de otros sitios por lo cual se puede conocer algunas conexiones tentativas del sitio con otros centros más grandes como Cnosos y, más alejados, Chania (Anderson 2016: 150; MacGillivray et al, 1989: 438-444).

\section{CONCLUSIÓN: ¿QUÉ NOS DICEN LOS SISTEMAS DE SELLADOS SOBRE LA REDISTRIBUCIÓN?}

De la larga exposición anterior, podemos señalar, citando a Weingarten (1989: 40), que: "no hay dos sitios minoicos que tengan estructuras de sellado idénticas". Esta afirmación es fundamental en nuestro estudio ya que hemos destacado algunas objeciones al modelo clásico de poder y economía en la isla.

La primera de ellas parte al analizar la isla de Creta como un conjunto. Ya, autores como Hamilakis (2002a) han advertido sobre la necesidad de desinsularizar los estudios sobre el pueblo minoico ya que entender al territorio como una unidad ocasiona que se considere que todas las regiones pertenecían a un mismo sistema general lo que lleva a ocultar las diversas diferencias particulares. Desde este punto de vista, hemos enfatizado que la unidad económica de Creta es una construcción historiográfica que se enmarca en una continuación de las ideas de Evans que hicieron eco en la obra de Finley, la cual, a su vez, estaba fuertemente influida por la información en las tablillas de Lineal B y en los descubrimientos en el Cercano Oriente.

En segundo lugar, desde el punto de vista arqueológico, los 'palacios' no contaban con la capacidad de almacenamiento suficiente para llevar a cabo una posterior redistribución que incluya a los pobladores cercanos o a los habitantes de esta estructura. Ante esto, se han establecido dos posibilidades: o bien las 'villas' formaban parte de subcentros de redistribución o bien el poder de los 'palacios' no era tal y estas estructuras en realidad eran otros centros de poder con menos parafernalia, pero con cierto control sobre sus territorios (Hitchcock y Preziosi, 1997).

En tercer lugar, los 'palacios' no parecen haber tenido tanto influencia en el intercambio como se suponía anteriormente ya que muchos de ellos se encuentran alejados de la costa o no cuentan con la evidencia material que los vincule como actores exclusivos o principales de los movimientos de bienes primarios y manufacturas tanto a nivel mediterráneo como regional. En este sentido, como mencionamos, han surgido nuevas aproximaciones que muestran una variedad de posibilidades para explicar las dinámicas políticas, económicas y culturales en la isla de Creta sin caer en la historiográficamente construida superioridad de Cnosos. Asimismo, también se han postulado una gran diversidad de modelos de intercambio cultural para explicar las interacciones en el marco del Mediterráneo Oriental.

Finalmente, nuestro aporte está dado por el análisis de los sellos. Ante esto, hemos destacado la gran diversidad de sistemas de sellados que existieron en Creta desde finales del periodo Protopalacial y durante el período Neopalacial. Teniendo en cuenta nuestra exposición, es pertinente preguntarnos hasta qué punto se puede hablar de redistribución en la isla de Creta durante el período Neopalacial si las estrategias administrativas y burocráticas variaron según las regiones ya que cada estructura monumental parece haber optado por una organización propia. ¿Es posible hablar de un estado que haya extendido su hegemonía sobre toda la isla de Creta? Evidentemente, la respuesta dependerá en gran parte por lo que se entienda como estado, pero desde el punto de vista de los sellos y su relación con la administración, nuestra respuesta tiene que ser negativa. ¿Estamos, entonces, ante estados regionales? Nuevamente, nos encontramos con una cuestión de definiciones que no podemos abarcar en este trabajo, pero tal vez sea necesario incorporar otras concepciones sobre las dinámicas sociopolíticas que no partan de categorías estancas. En este sentido, Hamilakis (2002b) ha propuesto considerar que las diferentes estructuras monumentales en Creta correspondían a grupos análogos a facciones que competían por el poder y que desarrollaron una parafernalia particular para expresarla. Desde una perspectiva similar, Driessen (2010) ha considerado la posibilidad de que nos encontremos ante 
households (casas o unidades domésticas) con mecanismos similares de competencia, pero con una importante dosis de solidaridad basada en el parentesco.

Estas dos posturas -entre otras- nos invitan a plantear nuevos caminos interpretativos y a establecer un puente con la antropología para buscar diferentes maneras de entender las relaciones de poder, de hegemonía $\mathrm{y}$, en este caso, de estrategias económicas. En futuros estudios exploraremos estas posibilidades.

Por lo pronto, resta agregar una cuestión más: en nuestro trabajo no estamos negando que no hayan existido ciertas estrategias redistributivas, sino más bien, consideramos que estas no fueron lo suficientemente amplias como para abarcar la totalidad de la economía ya sea en la redistribución de bienes primarios como en la producción de manufacturas. Sin embargo, es evidente que existieron prácticas redistributivas ya que los espacios de almacenaje, el sistema de sellados y las inscripciones en Lineal A y escritura Jeroglífica nos muestran un interés burocrático en controlar el ingreso y egreso de bienes. A esta estrategia se la podemos catalogar como "redistribución limitada" dado que tan solo buscaba abastecer algunas de las necesidades planteadas (Christakis 2011: 198) o "movilización” (Moody, 1987: 240) por la escasa cantidad de bienes involucrados ${ }^{8}$. Ante esto, la pregunta que se desprende es ¿̇redistribución limitada a qué? Ya en otra oportunidad (Cano Moreno, 2016: 211-218) hemos señalado la importancia de la religión en la sociedad minoica, sobre todo, en lo que se refiere al consumo conspicuo de parafernalia y a la preocupación iconográfica y material en hacer presente una diversidad de práctica cultuales. En este sentido, en próximos trabajos abordaremos esta problemática continuando los lineamientos aquí presentados y agregando la información iconográfica que presentaban los sellos y sellados cretenses.

\section{REFERENCIAS}

Adams, E. (2006). Social Strategies and Spatial Dynamics in Neopalatial Crete: An Analysis of the North-Central Area. American Journal of Archaeology ,110(1), 1-36.

Alexiou, S. (1987). Minoan Palaces as Centres of Trade and Manufacture. En N. Marinatos y R. Hägg (Eds.), The Function of the Minoan Palaces. Proceedings of the Fourth International Symposium at the Swedish Institute in Athens, 10-16 June. 1984 (pp. 251-253). Acta Instituti Atheniensis RegniSueciae Series in $4^{\circ}$, XXXV. Estocolmo: Paul Åström Forlag.

Anderson, E. (2016). Seals, Craft, and Community in Bronze Age Crete. Nueva York: Cambridge University Press.

Barjamovic, G. (en prensa). Interlocking Commercial Networks and the Infrastructure of Trade in Western Asia During the Bronze Age. En K. Kristiansen, T. Lindkvist y J. Myrdal (Eds.). Trade and Civilization in the PreModern World. Cambridge: Cambridge University Press.

Betancourt, P.P. (2002). Who Was in Charge of the Palaces? En J. Driessen, I. Schoep, y R. Laffineur (Eds.). Monuments of Minos. Rethinking the Minoan Palaces. Proceedings of an International Workshop held in Louvainla-Neuve, 2001. Aegaeum 23 (pp. 207-211). Lieja: Universidad de Lieja.

Betancourt, P.P. y Marinatos, N. (1997). The Minoan Villa. En Hägg, R. (Ed.). The Function of the "Minoan Villa". Proceedings of the Eighth International Symposium at the Swedish Institute at Athens, 6-8 June 1992. Svenska Institutet $i$ Athen Series in 4 $4^{\circ}$ XL (pp. 91-99). Estocolmo: Paul Åström Forlag.

Branigan, K. (1983). Craft Specialization in Minoan Crete. En O. Krzyszkowska y L. Nixon (Eds.). Minoan Society: proceedings of the Cambridge Colloquium (pp. 23-32). Bristol: Bristol Classical Press.

Branigan, K. (1988). Social Security and the State in Middle Bronze Age Crete. Aegaeum, 2, 11-16.

Branigan, K. (2001). Aspects of Minoan Urbanism. En K. Branigan (Ed.). Urbanism in the Aegean Bronze Age (pp. 38-50). Sheffield: Sheffield Academic Press Ltd.

Cano Moreno, J. (2016). Política, economía y religión en Creta Neopalacial. Hacia la construcción de una identidad de elite. En R. Flammini y J.M. Tebes. Interrelaciones e identidades culturales en el Cercano Oriente Antiguo (pp. 201-238). Ciudad Autónoma de Buenos Aires: IMHICIHU. 
Christakis, K. (1999). Pithoi and Food Storage in Neopalatial Crete: A Domestic Perspective. Food Technology in Its Social Context: Production, Processing and Storage. World Archaeology 31(1), 1-20.

Christakis, K. (2004). Palatial Economy and Storage in Late Bronze Age Knossos. En G. Cadogan, E. Hatzaki y A. Vasilakis (Eds.), Knossos: Palace, City, State (pp. 299-307). BSA Studies 12. Londres: British School at Athens.

Christakis, K. (2008). The Politics of Storage. Storage and Sociopolitical Complexity in Neopalatial Crete. Filadelfia: INSTAP Academic Press.

Christakis, K. (2011). Redistribution and Political Economies in Bronze Age Crete. Forum. Redistribution in Aegean Palatial Societies, American Journal of Archaeology , 115, 197-205.

Crowley, J. (2013). The Iconography of Aegean Seals. Aegaeum 34. Lieja: Université de Liège.

Dickinson, O. (2000) [1994]. La Edad del Bronce Egea. Madrid: Akal.

Dionisio, G., Jasink, A. y Weingarten, J. (2014). Minoan Cushion Seals. Innovation in Form, Style, and Use in Bronze Age Glyptic. Studia Archaeologica 196. Roma: L'Erma di Bretschneider.

Driessen, J. (2010). Spirit of Place. Minoan Houses as Major Actors. En D. Pullen (Ed.). Political Economies of the Aegean Bronze Age (pp. 35-65). Oxford: Oxbow Books.

Earle, T. (2011). Redistribution and the Political Economy: The Evolution of an Idea. Redistribution in Aegean Palatial Societies. American Journal of Archaeology, 115, pp. 237-244.

Evans, A. (1921-1936). The Palace of Minos: a Comparative Account of the Successive Stages of the Early Cretan Civilization as Illustred by the Discoveries at Knossos. 4 Vols. Londres: MacMillan and Co.

Finley, M. (1957). The Mycenaean Tablets and Economic History. Review of Economic History, 10, 128-141.

Galanakis, K. 2005. Minoan Glyptic: Typology, Deposits and Iconography from the Early Minoan Period to the Late Minoan IB Destruction in Crete. BAR International Series 1442. Oxford: Hadrian Books.

Hägg. R. y Marinatos, N. (Eds.). (1984). The Minoan Thalassocracy. Myth and Reality. Proceedings of the third international symposium at the Swedish Institute in Athens, 31 May-5 June 1982. Acta Instituti Atheniensis Regni Sueciae, series in 4o. Estocolmo: Paul Åström Forlag.

Hallager, E. (1996). The Minoan Roundel and Other Sealed Documents in the Neopalatial Linear A Administration. Aegaeum 14. Lieja: Université de Liège.

Hallager, E. (2001). Sealing without Seals - an Explanation. Working Paper no. 99-01. Centre for Cultural Research, University of Aarhus.

Halstead, P. (2011). Redistribution in Aegean Palatial Societies: Terminology, Scale and Significance. Forum. Redistribution in Aegean Palatial Societies, American Journal of Archaeology, 115, 229-235.

Hamilakis, Y. (Ed.). (2002a). The Labyrinth Revisited. Re-thinking the 'minoan' Archaeology. Oxford: Owbow Books. Hamilakis, Y. (2002b). Too many chiefs? Factional competition in Neopalatial Crete. En J. Driessen, I. Schoep, y R. Laffineur (Eds.). Monuments of Minos. Rethinking the Minoan Palaces. Proceedings of an International Workshop beld in Louvain-la-Neuve, 2001 (pp. 179-199). Aegaeum 23.

Hitchcock, L. y Preziosi. D. (1997). The Knossos Unexplored Mansionand the 'Villa-Anex Complex'. En R. Hägg (Ed.). The Function of the "Minoan Villa" (pp. 51-62). Estocolmo: Astrom Editions.

Karadimas, N. y Momigliano, N. (2004). On the Term 'Minoan' before Evans's Work in Crete (1894). SMEA, 46(2), 243-258.

Knapp, B. (1990). Ethnicity, Entrepreneurship, and Exchange: Mediterranean Inter-Island Relations in the Late Bronze Age. The Annual of the British School of Athens, 85, 115-153.

Knappett, C. (1999). Assessing a Polity in Protopalatial Crete: The Malia-Lasithi State. American Journal of Archaeology, 103(4), 615-639.

Knappett, C. y Nikolakopoulou, I. (2008). Colonialism without Colonies? A Bronze Age Case Study from Akrotiri, Thera. Hesperia: The Journal of the American School of Classical Studies at Athens, 77(1), 1-42.

Kopcke, G. (1987). The Cretan Palaces and Trade. En N. Marinatos y R. Hägg (Eds.). The Function of the Minoan Palaces. Proceedings of the Fourth International Symposium at the Swedish Institute in Athens, 10-16 June. 1984 (pp. 255-259). Acta Instituti Atheniensis RegniSueciae Series in $4^{\circ}, \mathrm{XXXV}$. Estocolmo: Paul Åström Forlag. 
Krzyszkowska, O. (2005). Aegean Seals: An Introduction. Bulletin of the Institute of Classical Studies. Supplement No. 85. Londres: University of London.

Lindgren, M. (1987). The Function of the Minoan Palaces-Myth and Reality. En N. Marinatos y R. Hägg(Eds.).The Function of the Minoan Palaces. Proceedings of the Fourth International Symposium at the Swedish Institute in Athens, 10-16 June. 1984 (pp. 39-42). Acta Instituti Atheniensis RegniSueciae Series in $4^{\circ}$, XXXV. Estocolmo: Paul Åström Forlag.

Marcelis, E. (1989). Quelques Réflexions à propos de l'organisation de l'artisanat minoen à l'époque des premiers et des seconds palais. En R. Laffineur (Ed.), Transition. Le Monde égéen du Bronze moyen au Bronze récent. Aegaeum 3 (pp. 29-34). Lieja: Université de Liège.

MacDonald, C., Hallager, E., y Niemeier, W.D. (Eds.) (2009). The Minoans in the Central, Eastern and Northern Aegean - New Evidence. Dinamarca: Monographs of the Danish Institute at Athens, vol. 8.

MacGillivray, A., Sackett, L.H., Driessen, J. y Smyth, D. (1989). Excavations at Palaikastro. British School of Athens, 84, 129-139.

MacGillivray, A. y Sackett, L. (2010). Palaikastro. E. Cline (Ed.) The Oxford Handbook of the Bronze Age Aegean (ca. 3000-1000 BC) (pp. 571-581). Nueva York y Oxford: Oxford University Press.

Marinatos, N. y Hägg, R. (Eds.). (1987). The Function of the Minoan Palaces. Proceedings of the Fourth International Symposium at the Swedish Institute in Athens, 10-16 June. 1984. Acta Instituti Atheniensis RegniSueciae Series in $4^{\circ}, \mathrm{XXXV}$. Estocolmo: Paul Åström Forlag.

McEnroe, J. (2011). Architecture of Minoan Crete: Constructing Identity in the Aegean Bronze Age. Austin: University of Texas Press.

Melas, M. (1988). Minoans Overseas: Alternative Models of Interpretation. Aegaeum 2, 47-69.

Michailidou, A. (1999). System of Weight and Social Relation of 'Private' Production in the Late Bronze Aegean. En A. Chaniotis (Ed.). From Minoan Farmers to Roman Traders: Sidelights on the Economy of Ancient Crete (pp.87-1099). Stuttgart: Steiner.

Molloy, B. (2012). Martial Minoans? War as Social Process, Practice and Event in Bronze Age Crete. The Annual of the British School of Athens, 107, 87-142.

Moody, J. (1987). The Minoan Palace as a Prestige Artifact. En N. Marinatos,y R. Hägg (Eds.). The Function of the Minoan Palaces. Proceedings of the Fourth International Symposium at the Swedish Institute in Athens, 10-16 June. 1984. Acta Instituti Atheniensis RegniSueciae Series in $4^{\circ}, X X X V$ (pp. 235-240). Estocolmo: Paul Åström Forlag.

Mountjoy, P.A. y Ponting, M.J. (2000). The Minoan Thalassocracy Reconsidered: provenance studies of LH IIA/LM IB pottery from Phylakopi, Ay. Irini and Athens. The Annual of British School at Athens, 95, 141-184.

Nakassis, D., Parkinson, W., y Galaty. M. (2011). Redistributive Economies from a Theoretical and Cross-Cultural Perspective. Forum. Redistribution in Aegean Palatial Societies, American Journal of Archaeology, 115, 174-188.

Parkinson, W. (2010). Beyond the Peer: Social Interaction and Political Evolution in the Bronze Age Aegean. En D. Pullen (Ed.), Political Economies of the Aegean Bronze Age (pp. 11-34).Oxford: Oxbow Books.

Platon, N. (1961). Chronologie de la Crète et des Cyclades à l'Age du Bronze. En G. Bersu y W. Dehn (Eds.), Bericht über den V. Internationalen Kongress für Vor-und Frühgeschichte, Hamburg vom 24. bis 30. August 1958 (pp. 671-676). Berlín: Mann.

Polanyi, K. (1944). The Great Transformation. Boston: Beacon Press.

Pullen, D. (Ed.). (2010). Political Economies of the Aegean Bronze Age. Oxford: Oxbow Books.

Hawes, H.B., Williams, B., Seager, R. y Hall, E. (1908). Gournia: Vasiliki and other prehistoric sites on the isthmus of Hierapetra, Crete; excavations of the Wells-Houston-Cramp expeditions, 1901, 1903, 1904. Filadelfia: The American Exploration Society.

Hägg, R. (Ed.). (1997). The Function of the "Minoan Villa". Proceedings of the Eighth International Symposium at the Swedish Institute at Athens, 6-8 June, 1992. Svenska nstitutet i Athen Series in $4^{\circ}$, XL. Estocolmo: Paul Åström Forlag. 
Renfrew, C. (1972). The Emergence of Civilisation. The Cyclades and the Aegean in the third millennium BC. Londres: Methuen.

Rehak, P. y Younger, J. (1998). Review of Aegean Prehistory VII: Neopalatial, Final Palatial and Postpalatial Crete. American Journal of Archaeology, 102(1), 91-173.

Rehak, P. y Younger, J. (2010 [2008]). Minoan Culture: Religion, Burial Customs, and Administration. En C.W. Shelmerdine, (ed.), The Cambridge Companion to the Aegean Bronze Age (pp. 165-185). Nueva York: Cambridge University Press.

Schoep, I. (1999a). Tablets and Territories? Reconstructing Late Minoan IB Political Geography through Undeciphered Documents. American Journal of Archaeology, 103(2), 201-221.

Schoep, I. (1999b). The Origins of Writing and Administration in Crete. Oxford Journal of Archaeology, 18(3), 265-276.

Schoep, I. (2001). Managing the Hinterland: The Rural Concerns of Urban Administration. En K. Branigan (Ed.), Urbanism in the Aegean Bronze Age (pp. 87-102). Londres: Sheffield Academic Press.

Schoep, I. (2002). The state of the Minoan palaces or the Minoan palace-state. En J. Driessen, I. Schoep, R. Laffineur (Eds.), Monuments of Minos. Rethinking the Minoan Palaces. Proceedings of an International Workshop held in Louvain-la-Neuve, 2001 (pp. 15-33). Aegaeum 23. Lieja: Universidad de Lieja.

Schoep, I. (2010). The Minoan 'Palace-Temple' Reconsidered: A Critical Assessment of the Spatial Concentration of Political, Religious and Economic Power in Bronze Age Crete. Journal of Mediterranean Archaeology, 23(2), 219-244.

Shelmerdine, C.W. (2010 [2008]). Background, Sources and Methods. En C.W. Shelmerdine, (ed.), The Cambridge Companion to the Aegean Bronze Age (pp. 1-18). Nueva York: Cambridge University Press.

Soles, J. (1991). The Gournia Palace. American Journal of Archaeology, 95(1), 17-78.

Starr, C.G. (1955). The Myth of the Minoan Thalassocracy. Historia: Zeitschrift fur Alte Geschichte, 3, 282-291.

Vavouranakis, G. (2007). Palatial Style Architecture and Power in Bronze Age Crete. En S. Antoniadou y A. Pace (Eds). Mediterranean Crossroads (pp.263-289). Atenas: Oxbow Books.

Watrous, L.V. y Heimroth, A. (2011). Household Industries of Late Minoan IB Gournia and the Socioeconomic Status of the Town. ITETA: The Archaeology of Houses and Households in Ancient Crete (pp.199-212). Hesperia Supplements 44.

Watrous, L.V., Buell, D.M., McEnroe, J., Younger, J., Turner, L.A., Kunkel, B.S., Glowacki, K., Gallimore, S., Smith, A., Pantou, P., Chapin, A. y Margaritis, E. (2015). Excavations at Gournia, 2010-2012. Hesperia, 84(3), 397-465.

Weingarten, J. (1986). The Sealing Structures of Minoan Crete I: MM II Phaistos to the Destruction of the Palace of Knossos. Part 1: The Evidence Until the LM IB Destructions. Oxford Journal of Archaeology, 5(3), 279-298.

Weingarten, J. (1989). New and Old Elements in the Seals and Sealings of the Temple Repository, Knossos. En R. Laffineur (Ed.) Transition. Le Monde égéen du Bronze moyen au Bronze récent (pp. 39-52). Aegaeum 3. Lieja: Université de Liège.

Weingarten, J. (1990a). Three Upheavals in Minoan Sealing Administration: Evidence for Radical Change. En T. Palaima (Ed.), Aegean Seals, Sealings and Administration. Proceedings of the NEH-Dickson Conference of the Program in Aegean Scripts and Prehistory of the Department of Classics, University of Texas at Austin, January 11-13, 1989 (pp. 105-120). Aegaeum 5. Lieja: Université of Liège.

Weingarten, J. (1990b). The Sealing Structure of Karahoyuk and Some Administrative Links with Phaistos on Crete. Oriens Antiquus, XXIX, 63-95.

Weingarten, J. (1987). Seal Use at LM I B Ayia Triada: a Minoan Elite in Action I. Administrative Considerations. Kadmos, 26, 1-43.

Weingarten, J. y Webb, J. (2012). Seals and seal use: markers of social, political and economic transformations on two islands. En G. Cadogan, M. Iacovou, K. Kopaka, y J. Whitley (Eds.), Parallel Lives: Ancient Island Societies in Crete and Cyprus (pp.85-104). British School at Athens Studies 20. Londres: British School at Athens. 
Whitelaw, T. (en prensa). Feeding Knossos: exploring economic and logistical implications of urbanism on Prehistoric Crete. En D. Garcia, R. Orgeolet, M. Pomadiere y J. Zurbach (Eds.), The country in the city. Oxford: Archaeopress.

Wiener, M. (1990). The Isles of Crete? The Minoan Thalassocracy Revisited. En D. A. Hardy, C. G. Doumas, J. A. Sakellarakis, y P. M. Warren (Eds.). Thera and the Aegean World III, Volumen One: Archaeology (pp. 128-161). Londres: The Thera Foundation.

Wiener, M. (1991). The Nature and Control of Minoan Foreign Trade. En N. H. Gale (Ed.), Bronze Age Trade in the Mediterranean. Papers Presented at the Conference held Rewley House, Oxford in December 1989 (pp. 325-351). Jonsered: Paul Åström Forlag.

Wiener, M. (2015). Dating the Theran Eruption: Archaeological Science Versus Nonsense Science. En T.E. Levy, T. Schneider y W.H.C. Propp (eds.). Israel's Exodus in Transdisciplinary Perspective. Quantitative Methods in the Humanities and Social Sciences (pp. 131-143). New York: Springer International Publishing.

\section{Notas}

1 La cronología para Creta en la Edad de Bronce es un tema complejo que ha sido fruto de diversos trabajos, pero para que el lector pueda comprender los términos utilizados señalamos que en nuestro campo hay tres nomenclaturas: la cerámica que divide el marco temporal entre Temprano, Medio y Reciente con sus divisiones en números latinos y sus subfases en letras. Sin embargo, N. Platon (1961) remarcó que las fases cerámicas no se correspondían con el desarrollo de las estructuras monumentales por lo cual utilizó la arquitectura como una variable cronológica más fiable. Así, dividió la historia cretense en Prepalacial, Protopalacial, Neopalacial, Palacial Final y Postpalacial que en castellano ibérico fue traducido como Prepalacial, Primeros Palacios, Segundos Palacios, Terceros Palacios y Postpalacial. Esta última periodización no es utilizada más que en algunos estudios de España. Finalmente, en cuanto a las fechas absolutas, es difícil encontrar un acuerdo entre las cronologías bajas, medias y altas dado que cada autor suele seguir las fechas relativas de su sitio arqueológico. Aun así, podemos comprender al período Neopalacial, grosso modo, entre el 1700 y el 1450 a.C. Para más información véase Shelmerdine (2008, 3-14), Dickinson (1994, 9-22), como lecturas introductorias y Wiener (2015, 131-143) para un resumen de las complicaciones existentes para calibrar las dataciones de este período.

2 Una gran cantidad de términos utilizados en el estudio de la cultura minoica son construcciones historiográficas nacidas a principios del siglo XX y generalmente repetidas acríticamente. En nuestro caso, utilizaremos las comillas simples para destacar que cuando mencionemos estos términos no lo haremos de manera literal sino señalando que son válidos para llegar a un común entendimiento dado que ningún otro término ha sido aceptado de manera mayoritario. Para el caso del término 'palacio' puede verse de manera introductoria a Schoep (2010a). En cuanto nos refiramos al caso micénico, obviaremos las comillas simples dado que en ese caso sí existían monarcas (wanakes) que habitaban estos edificios.

3 Durante la Edad de Bronce, Creta tuvo 5 sistemas de escritura: 3 difundidos por toda la isla (el [mal]llamado jeroglífico, el Lineal A y el Lineal B) y otros dos que constituyen unica (la "escritura de Arcanes" y los símbolos del Disco de Festo). En el período Neopalacial encontramos en uso la escritura jeroglífica y el Lineal A, las cuales, como ha señalado Schoep (1999b) no están estandarizadas y no siempre se encuentran en contextos palaciales, tal y como sucede con los sellos. Si bien se sabe que ambos sistemas son silábicos no es posible conocer el lenguaje que transmiten y tan sólo se conoce el valor de algunos símbolos por su presencia en las tablillas de Lineal B que corresponden al período Micénico y que están escritas en una forma silábica del griego. Para más información, véase: Schoep (1999a y b) y Rehak y Younger (1998 y 2008).

4 Utilizamos el término no como reemplazo de 'palacio', sino como elemento arquitectónico tal y como lo utiliza McEnroe (2011: 84-86).

5 Tal vez sea necesario aclarar que este modelo actualmente también es cuestionado en los estudios económicos del Cercano Oriente. Cfr. Yoffee y Barjamovich (en prensa).

6 Para los periodos anteriores y posteriores al mencionado los contextos arqueológicos en donde se puede encontrar estos objetos también incluyen diferentes rangos de tumbas.

7 Según Krzyszkowska 2005: 171, son 975.

8 Respecto a este punto, este término nos parece muy vago ya que la redistribución incluye a la movilización y como concepto es demasiado descriptivo y no ahonda en los mecanismos de dicho movimiento de bienes. 